\title{
Effect of intensive care unit environment on in-hospital delirium after cardiac surgery
}

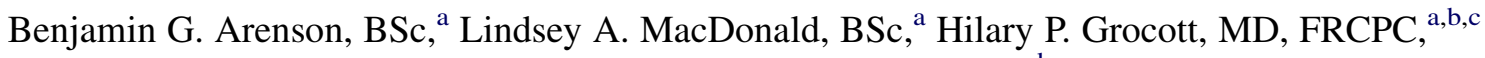 \\ Brett M. Hiebert, MSc, ${ }^{\mathrm{c}}$ and Rakesh C. Arora, MD, PhD, FRCSC ${ }^{\mathrm{a}, \mathrm{b}, \mathrm{c}}$
}

\begin{abstract}
Objectives: The etiology of postcardiac surgery delirium is complex. Our primary objective was to determine the effect of the postoperative environment on the prevalence of delirium by examining the in-hospital delirium rates in 2 postoperative intensive care units with differing physical infrastructure. We further sought to identify other risk factors associated with in-hospital delirium.
\end{abstract}

\begin{abstract}
Methods: The rates of postoperative delirium were retrospectively examined in consecutive cardiac surgery patients during 2 separate 6-month periods. Environment 1 was characterized by a lack of physical barriers between bed spaces and was windowless, and environment 2 consisted of private rooms with physical barriers for each patient and with wall-to-wall exterior windows. Univariate and multivariate analyses to determine the risk factors associated with in-hospital delirium, including the effect of environment, were undertaken.
\end{abstract}

Results: Of the 1010 patients studied, 148 (14.7\%) experienced in-hospital delirium after cardiac surgery. The prevalence of delirium was not significantly different between environments 1 and $2(16.1 \%$ vs $13.5 \% ; P=.25)$. However, in patients younger than 65 years, the proportion of intensive care unit days on which delirium occurred was greater in environment 1 than in environment $2(5.4 \%$ vs $1.7 \% ; P=.006)$. Postoperative stroke or transient ischemic attack, mechanical ventilation longer than 24 hours, age 65 years or older, concomitant coronary artery bypass grafting and valve surgery, prehospital admission benzodiazepine use, a requirement for any postoperative blood product transfusion, and postoperative renal insufficiency were identified as risk factors.

Conclusions: The intensive care unit environment did not have a significant effect on the overall prevalence of delirium. However, that does not preclude the possibility that the intensive care unit environment might interact with other factors, such as age, in a complex manner. Attempts to reduce delirium by adjusting the intensive care unit environment alone will likely not be sufficient, and instead will require a more comprehensive multimodal approach. (J Thorac Cardiovasc Surg 2013;146:172-8)

Delirium is a symptomatic manifestation of acute brain dysfunction during which patients can exhibit behavior ranging from unresponsive and withdrawn to agitated and combative. It is a frequent complication after cardiac surgery and has been associated with prolonged hospital stay, ${ }^{1}$ increased hospital costs, ${ }^{2}$ and an increased risk of mortality. ${ }^{3}$ The emerging trend of older patients with greater levels of comorbidity burden being referred for cardiac surgery ${ }^{4}$ will likely exacerbate this issue, because both these characteristics are associated with an increased risk of delirium. ${ }^{5}$

From the Department of Anesthesia and Perioperative Medicine, ${ }^{\mathrm{a}}$ and Department of Surgery, ${ }^{\mathrm{b}}$ University of Manitoba; and Cardiac Sciences Program, ${ }^{\mathrm{c}}$ Winnipeg Regional Health Authority, Winnipeg, Manitoba, Canada.

This study was supported by an internal grant from the Department of Anesthesia and Perioperative Medicine, University of Manitoba Faculty of Medicine, Winnipeg, Manitoba, Canada.

Disclosures: Authors have nothing to disclose with regard to commercial support.

Received for publication Aug 27, 2012; revisions received Nov 19, 2012; accepted for publication Dec 11, 2012; available ahead of print Jan 14, 2013.

Address for reprints: Rakesh C. Arora, MD, PhD, FRCSC, CR 3009 St. Boniface Hospital, Winnipeg, Manitoba R2H 2A6, Canada (E-mail: rakeshcarora@gmail.com). 0022-5223/\$36.00

Copyright (c) 2013 by The American Association for Thoracic Surgery http://dx.doi.org/10.1016/j.jtcvs.2012.12.042
The development of reliable systematic screening tools such as the Confusion Assessment Method (CAM) ${ }^{6}$ and $\mathrm{CAM}$ for the intensive care unit (CAM-ICU) ${ }^{7}$ has helped in both aiding diagnosis and facilitating the identification of potential risk factors associated with delirium. The CAM and CAM-ICU are validated screening instruments for delirium as defined by the "Diagnostic Statistical Manual of Psychiatric Disorders," with high specificity and sensitivity and low inter-rater variability. ${ }^{6,7}$ The CAM-ICU is administered in conjunction with the Richmond Agitation-Sedation Scale (RASS), ${ }^{8}$ a scoring scale used for the assessment of a patient's level of sedation and agitation.

Among the risk factors associated with delirium are those related to the hospital and ICU environment, such as high levels of background noise and an absence of natural light. Both of these factors have been found to disrupt normal sleep-wake cycles, resulting in patients who are sleep deprived and at a greater risk of delirium. ${ }^{9}$ Although previous studies have examined the association between the ICU environment and delirium, ${ }^{9,10}$ none have investigated this in a cardiac surgery setting. At our center, the planned opening of a new postoperative cardiac surgery ICU, with 


\section{Abbreviations and Acronyms \\ $\mathrm{CABG}=$ coronary artery bypass grafting \\ CAM = Confusion Assessment Method \\ $\mathrm{CAM}-\mathrm{ICU}=\mathrm{CAM}$ for the intensive care unit \\ E1 = environment 1 \\ E2 = environment 2 \\ ICU = intensive care unit \\ IQR = interquartile range \\ RASS $=$ Richmond Agitation-Sedation Scale \\ TIA $=$ transient ischemic attack}

a modernized physical infrastructure, provided us with a unique opportunity to study the effect that the ICU environment could have on the prevalence of delirium after cardiac surgery. Our previous postoperative cardiac surgery ICU was a dedicated specialized unit with a 24-hour consultant providing in-house care ${ }^{11}$ and was located in the center of the main hospital building adjacent to the postanesthetic care unit and operating rooms. This environment was characterized by a lack of physical barriers between the bed spaces' exposure to high "traffic" noise, and was windowless (Figure 1, $A$ ). The newly created ICU encompasses an entire floor in a separate building with less exposure to "traffic" noise and consists of private rooms with physical barriers for each patient, wall-to-wall exterior windows, and access to a visible clock (Figure 1, B).

The aim of our study was to investigate the effect of the ICU environment on the prevalence of in-hospital delirium after cardiac surgery. A secondary aim was to identify the risk factors for in-hospital delirium in our contemporary cardiac surgical population.

\section{METHODS}

\section{Study Population}

A retrospective analysis was undertaken of all consecutive patients who had undergone cardiac surgery at our center from May to October in 2 consecutive years (2010 and 2011). Two cohorts of patients were examined: those patients admitted to the "old" ICU environment (E1) from May 1, 2010, to October 31, 2010, and those admitted to the "new" ICU environment (E2) from May 1, 2011, to October 31, 2011. The use of E2 followed the opening of the new cardiac surgery ICU in April 2011. During these two periods, no formal changes were made to the protocols related to sedation, analgesia, and delirium prophylaxis and treatment (which were left under the purview of the healthcare team responsible for the patient). A consistent study period for each ICU environment (May to October) was chosen to use periods in which the maximal amount of daylight occurs in Manitoba. The patients who presented with a pre-existing structural brain disease (ie, previous brain tumor, subarachnoid hemorrhage, or seizure disorder), surgery for cardiac trauma, pericardiectomy, thoracic endovascular aortic repair, extracorporeal membrane oxygenation, left ventricular lead placement, had died in hospital, or were missing all CAM and CAM-ICU data from the ICU or in-patient ward were excluded from the final analysis. The University of Manitoba research ethics board approved the study, and the requirement for patient informed consent was waived.

\section{Delirium Assessment}

Beginning in March 2010, as a part of the standard care at our center, all postoperative cardiac surgery patients were assessed for delirium routinely using the CAM-ICU (at least every 8 hours) and RASS (at least every 4 hours) during their stay in the ICU and using the CAM (at least every 8 hours) on the in-patient ward. The CAM and CAM-ICU both assess 4 features of delirium: acute onset or fluctuating course, inattention, disorganized thinking, and altered level of consciousness. A positive diagnosis of delirium results from the first 2 features and either feature 3 or 4 . The determination of the patient's RASS is required for the assessment of the testability of the sedated patient and for differentiation between hypo- and hyperactive delirium. The RASS is a 10-point scale ranging from -5 to +4 , with scores of -1 to -5 for increasing levels of sedation, a score of 0 to denote a calm and alert state, and scores of +1 to +4 for increasing levels of anxiety or agitation. Coma is defined as a RASS score of -4 (responsive to physical but not verbal stimulus) or -5 (unresponsive to verbal and physical stimulus). Therefore, the diagnosis of delirium requires a RASS score of -3 or greater. Delirium data were collected from the assessments made from postoperative day 1 to 10 in either the ICU or in-patient ward. The patients were classified as having a day of delirium if delirium was detected in at least 1 assessment during the 24-hour period of a given postoperative day.

Ambient and/or artificial light levels were measured in each ICU environment recorded at 12:00 PM for 3 consecutive days in the south-facing area of each unit (Heavy Duty Light Meter with PC Interface, model 407026; Extech Instruments, Nashua, NH) and reported as the mean \pm standard deviation. During both ICU environment epochs, the same balanced anesthesia technique was used with up to $1.5 \mathrm{mg} / \mathrm{kg}$ propofol for induction of anesthesia, and maintenance including sufentanil $(\leq 1 \mu \mathrm{g} / \mathrm{kg})$, midazolam (0.01-0.02 mg/kg), and sevoflurane (1.0 MAC). For patients requiring mechanical ventilation in the ICU, sedation was titrated to target a RASS of -2 to 0 , where possible. Sedation and analgesic agents consisted of 1 or more of propofol, midazolam, and/or opioids. The preoperative, intraoperative, and postoperative variables were prospectively collected from the medical chart for each patient.

\section{Statistical Analysis}

The univariate and multivariate risk factors for in-hospital delirium after cardiac surgery were determined for the entire study population. Categorical variables were compared using the chi-square and Fisher exact tests; continuous variables were compared using the Mann-Whitney $U$ and $t$ tests. A final multivariate logistic regression model was developed through the use of a stepwise selection process considering all univariate risk factors with $P<.20$. Risk factors with $P<.05$ were considered statistically significant. Model discrimination was assessed by calculating the area under the receiver operating characteristic curve and its corresponding 95\% confidence interval. Goodness of fit for the model was determined by calculating the Hosmer-Lemeshow test statistic, with $P>.05$ indicating an appropriate fit. All statistical analyses were performed using SAS, version 9.2 (SAS Institute, Cary, NC).

The effect of E2 was included among the potential risk factors in the univariate and multivariate analyses. Covariate comparisons between E1 and E2 were made to identify the differences between the 2 populations. The proportion of ICU days with a delirium event was also compared between the ICU environments. These comparisons were also stratified by age to determine whether the ICU environment affected delirium in different subpopulations.

\section{RESULTS}

\section{Delirium Prevalence}

The final study population after all exclusions $(\mathrm{n}=92)$ consisted of 1010 cardiac surgery patients during the 2 


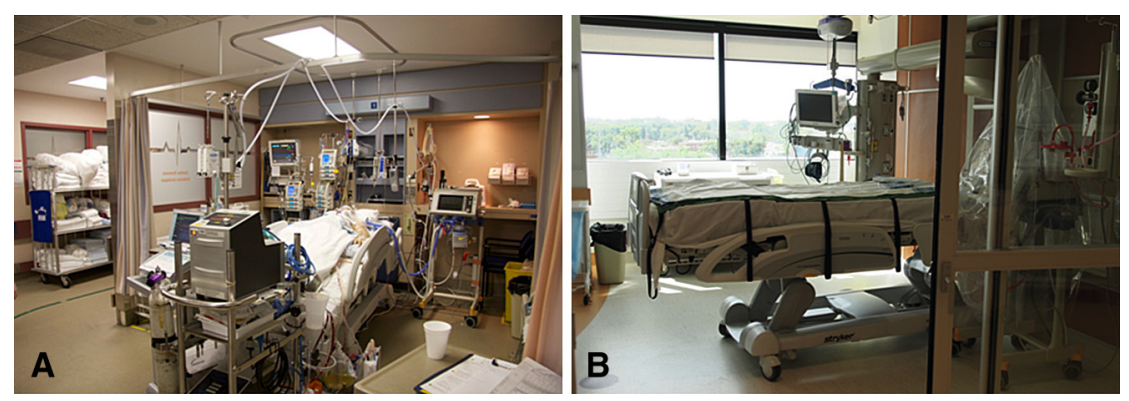

FIGURE 1. A, Photograph of environment 1 (E1) intensive care unit. B, Photograph of environment 2 (E2) intensive care unit.

separate 6-month periods. Of these patients, $646(63.9 \%)$ had undergone an isolated CABG procedure, 147 (14.6\%) an isolated valve procedure, $94(9.3 \%)$ a combined CABG and valve procedure, and $123(12.2 \%)$ other cardiac procedures. Approximately $30.4 \%$ of the patients were extubated immediately postoperatively before leaving the operating room. Most patients were men $(71.7 \%)$, and the average age was $65.5 \pm 11.6$ years.

The overall prevalence of any in-hospital delirium after cardiac surgery was $14.7 \%$. The prevalence of delirium among patients younger than 65 years and those 65 years or older was $6.2 \%$ and $21.4 \%$, respectively. Of the 148 delirious patients, $60(40.8 \%)$ had delirium only in the ICU, $53(36.1 \%)$ had delirium only on the hospital ward, and $34(23.1 \%)$ had a delirium event in both the ICU and the hospital ward. Of those experiencing delirium, the duration was 1 day for $46.8 \%, 2$ days for $21.8 \%, 3$ days for $12.2 \%$, and 4 or more days for $14.1 \%$. The length of stay in the ICU and the postoperative hospital stay was significantly longer for those patients with delirium than for those without delirium (median, 2.9 days; interquartile range [IQR], 1.7-5.1 days, vs median, 1.0 days; IQR, 0.9-2.0 days; $P<.001$ and median, 10 days; IQR, 7-16 days, vs median, 6; IQR, 5-8 days; $P<.001$, respectively).

\section{Role of ICU Environment}

No significant difference was found in the prevalence of in-hospital delirium between the 2 ICU environments (E1 and E2), with a rate of $16.1 \%$ and $13.5 \%$, respectively $(P=.25$; Figure 2). Similarly, no significant difference was found in the prevalence of ICU delirium, with a rate for E1 and $\mathrm{E} 2$ of $9.8 \%$ and $9.2 \%$, respectively $(P=.71)$. The median interval to the initial delirium onset was postoperative day 3 for both E1 and E2 (Figure 3). The initial onset of delirium was postoperative day 2 and later for $74 \%$ and $72 \%$ of delirious patients in E1 and E2, respectively. The length of stay in the ICU was significantly different between E1 and E2 (median, 1.0 days; IQR, 0.9-2.0 days, vs median, 1.6 days; IQR, 0.9-2.8 days; $P<.001$ ). The light levels in E1 and E2 were not significantly different, with a value of $535 \pm 75$ lux and $656 \pm 280$ lux, respectively $(P=.51)$.
However, the light source in E1 was exclusively fluorescent lighting and that in E2 was a mixture of natural daylight and fluorescent lighting. Covariate comparisons between E1 and E2 identified significant differences between the 2 populations (Table 1) and the potential influential interactions were tested in the final multivariate model. In patients younger than 65 years, the proportion of ICU days on which delirium occurred was significantly greater in E1 than in E2 $(5.4 \%$ vs $1.7 \% ; P=.006)$. In contrast, in patients aged 65 years and older, no differences were found in the proportion of ICU days on which delirium occurred in E1 compared with E2 (10.8\% vs $13.7 \% ; P=.099)$.

\section{Risk Factors Associated With Delirium}

The univariate analysis revealed significant variables (Table 2) that were included in the stepwise selection logistic regression analysis. Seven variables were identified as independent risk factors associated with in-hospital delirium after cardiac surgery (Table 3): postoperative stroke or transient ischemic attack (TIA), mechanical ventilation longer than 24 hours, age 65 years or older, concomitant CABG and valve surgery, prehospital admission benzodiazepine use, a requirement for any postoperative blood product transfusion, and postoperative renal insufficiency. The calculated area under the curve for our multivariate model

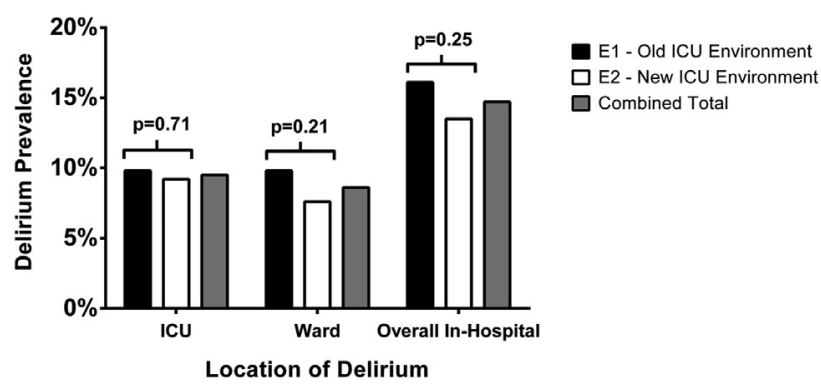

FIGURE 2. Prevalence of in-hospital delirium after cardiac surgery according to intensive care unit $(I C U)$ environment. Patients were admitted to environment 1 (E1) from May to October 2010 and to environment 2 (E2) from May to October 2011. Prevalence of delirium was not significantly different between E1 and E2. 


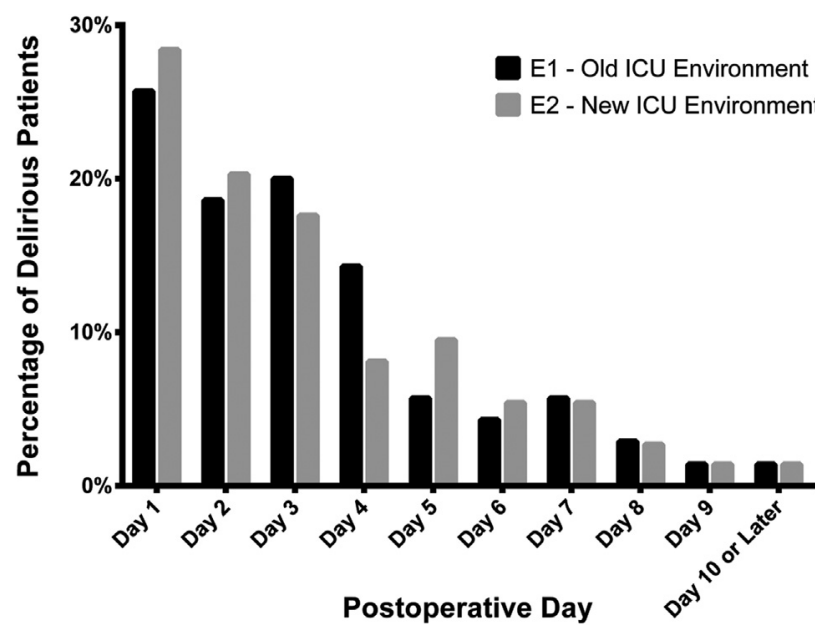

FIGURE 3. Initial onset of in-hospital delirium after cardiac surgery by postoperative day. $E 1$, Environment 1 ; $I C U$, intensive care unit; $E 2$, environment 2 .

was 0.794 (95\% confidence interval, $0.755-0.832)$, with a Hosmer-Lemeshow test statistic of $P=.3774$.

\section{DISCUSSION}

The present study represents one of the largest samples of cardiac surgery patients undergoing systematic screening for delirium. This large sample size ensured that the effect of the ICU environment on the overall prevalence of delirium would not be masked by a lack of statistical power. Furthermore, it is one of the few studies that investigated delirium in both the ICU and the hospital ward using a systemic screening tool.

In the present study of 1010 cardiac surgery patients, the overall prevalence of delirium was $14.7 \%$, with a marked

TABLE 1. Differences between patient populations in E1 and E2 ICU

\begin{tabular}{lccr}
\hline \multicolumn{1}{c}{ Risk factor } & E1 $(\mathbf{n}=\mathbf{4 5 4})$ & E2 $(\mathbf{n}=\mathbf{5 5 6})$ & $\boldsymbol{P}$ value \\
\hline Preoperative characteristics & & & \\
Smoking (previous) & $291(64.5 \%)$ & $307(55.8 \%)$ & .0052 \\
Albumin & $39(35-42)$ & $37(34-40)$ & $<.0001$ \\
Hemoglobin & $135(121-144)$ & $137(126-148)$ & .0123 \\
Anemia $(<120 \mathrm{~g} / \mathrm{L})$ & $114(25.1 \%)$ & $110(19.8 \%)$ & .0427 \\
HbA1c $(\%$ of total Hb) & $5.8(5.4-6.8)$ & $6.2(5.8-7.1)$ & $<.0001$ \\
Intraoperative characteristics & & & \\
Isolated valve procedure & $50(10.7 \%)$ & $98(17.5 \%)$ & .0020 \\
Fentanyl & $98(21.6 \%)$ & $77(13.9 \%)$ & .0014 \\
Morphine & $159(35.1 \%)$ & $133(24.0 \%)$ & .0001 \\
Neostigmine & $120(26.5 \%)$ & $116(21.0 \%)$ & .0400 \\
Sufentanil & $365(80.9 \%)$ & $484(87.1 \%)$ & .0079 \\
Antifibrinolytic (TXA) & $375(83.2 \%)$ & $428(77.0 \%)$ & .0154 \\
Lowest hemoglobin & $86(73-99)$ & $88(76-100)$ & .0441 \\
Postoperative characteristics & & & \\
Lowest sodium & $133(131-135)$ & $134(131-135)$ & .0376 \\
\hline Daprent
\end{tabular}

Data presented as mean \pm standard deviation or median (25th percentile-75th percentile). E1, Environment 1 ("old" ICU); E2, environment 2 ("new" ICU); ICU, intensive care unit; HbAlc, glycated hemoglobin; $H b$, hemoglobin; TXA, tranexamic acid. increase in prevalence in patients aged 65 years or older. These rates are both well within the range of $3 \%$ to $52 \%$ reported in a recent review of cardiac surgery studies. ${ }^{12}$ The present study is unique in its aim to examine the effect of physically modifying the postoperative environment on the prevalence of delirium in a cardiac surgery setting. In this population, the prevalence of in-hospital delirium after cardiac surgery did not vary significantly between E1 and E2, with a rate of $16.1 \%$ and $13.5 \%$, respectively. This suggests that the postcardiac surgical ICU environment either had a limited effect on the prevalence of delirium or perhaps was muted by influential differences between the 2 patient populations.

We identified postoperative stroke or TIA, mechanical ventilation longer than 24 hours, age 65 years or older, concomitant CABG and valve surgery, prehospital admission benzodiazepine use, any postoperative blood product transfusion, and postoperative renal insufficiency as significant risk factors associated with the development of in-hospital delirium after cardiac surgery.

In the present study, the onset of stroke or TIA in the postoperative period was the strongest risk factor for cardiac surgery-associated delirium, just as other investigators have previously found. ${ }^{5}$ It is unclear whether the onset of stroke or TIA and delirium postoperatively is causally related or whether the 2 events are both symptomatic manifestations of poor overall cerebral dysfunction. ${ }^{13}$

Prolonged mechanical ventilation was another strong risk factor associated with in-hospital delirium in this cardiac surgery population. When considering only the initial onset of delirium, mechanical ventilation for longer than 24 hours appeared to have a combined effect on the development of postoperative delirium. That is, cardiac surgery patients were more likely to become delirious while mechanically ventilated for such extended periods and also more likely to experience delirium after extubation following these periods. It is difficult to ascertain the exact causal relationship between prolonged mechanical ventilation and delirium. Evidence has suggested that prolonged mechanical ventilation causes delirium, ${ }^{14}$ and, conversely, that delirium leads to prolonged mechanical ventilation. ${ }^{15}$ Certainly, the sedative level and the clearance of drugs secondary to renal and/or hepatic dysfunction could play a role. However, in our population, those patients mechanically ventilated for longer than 24 hours had a median and mean RASS score of 0 and -1.2 , respectively. This suggests that the sedation level was not influencing the development of delirium in these patients.

Age 65 years and older was an independent risk factor associated with in-hospital delirium, a finding supported by numerous other studies. ${ }^{14-16}$ Furthermore, although the ICU environment did not exclusively affect the prevalence rates of delirium, we found preliminary evidence to suggest that the ICU environment could have a heterogeneous effect on delirium between older and younger patients. This might 
TABLE 2. Univariate analyses of potential risk factors associated with in-hospital delirium after cardiac surgery

\begin{tabular}{|c|c|c|c|}
\hline \multirow[b]{2}{*}{ Variable } & \multicolumn{2}{|c|}{ Hospital delirium } & \multirow[b]{2}{*}{$P$ value } \\
\hline & No $(n=862)$ & Yes $(n=148)$ & \\
\hline \multicolumn{4}{|l|}{ Preoperative characteristics } \\
\hline Age $(y)$ & $64.5 \pm 11.7$ & $71.5 \pm 8.8$ & $<.0001$ \\
\hline Age $\geq 65$ y & $446(50.9 \%)$ & $123(80.9 \%)$ & $<.0001$ \\
\hline Chronic obstructive pulmonary disease & $71(8.1 \%)$ & $20(13.2 \%)$ & .0444 \\
\hline Preoperative renal insufficiency (creatinine $>176 \mathrm{mmol} / \mathrm{L}$ ) & $31(3.5 \%)$ & $11(7.2 \%)$ & .0335 \\
\hline Creatinine & $84(71-99)$ & $89(74-111)$ & .0049 \\
\hline Albumin & $38(35-41)$ & $37(34-40)$ & .0379 \\
\hline Hemoglobin & $136(125-147)$ & $134(119-143)$ & $<.0001$ \\
\hline Anemia $(<120 \mathrm{~g} / \mathrm{L})$ & $184(21.0 \%)$ & $45(29.6 \%)$ & .0187 \\
\hline Benzodiazepine use (prehospital admission)* & $59(6.8 \%)$ & $16(10.6 \%)$ & .0998 \\
\hline \multicolumn{4}{|l|}{ Intraoperative characteristics } \\
\hline Operation type & & & .0007 \\
\hline Emergent & $53(6.1 \%)$ & $23(15.1 \%)$ & \\
\hline Urgent & $155(17.8 \%)$ & $29(19.1 \%)$ & \\
\hline Elective & $664(76.2 \%)$ & $100(65.8 \%)$ & \\
\hline Isolated valve procedure & $134(15.6 \%)$ & $13(8.8 \%)$ & .0312 \\
\hline $\mathrm{CABG}$ and valve procedure & $63(7.3 \%)$ & $31(21.0 \%)$ & $<.0001$ \\
\hline Deep hypothermic cardiac arrest & $8(0.9 \%)$ & $6(4.0 \%)$ & .0029 \\
\hline Redo sternotomy & $39(4.5 \%)$ & $15(9.9 \%)$ & .0059 \\
\hline Pump time & $95(72-130)$ & $117.5(88-153)$ & $<.0001$ \\
\hline Pump time $\geq 120 \mathrm{~min}$ & $271(30.9 \%)$ & $71(46.7 \%)$ & .0001 \\
\hline Desflurane & $3(0.3 \%)$ & $3(2.0 \%)$ & .0457 \\
\hline Glycopyrrolate & $244(27.8 \%)$ & $27(17.8 \%)$ & .0084 \\
\hline Midazolam & $683(78.3 \%)$ & $104(68.4 \%)$ & .0075 \\
\hline Neostigmine & $213(24.4 \%)$ & $25(16.5 \%)$ & .0316 \\
\hline Extubation in operating room & $280(32.0 \%)$ & $27(17.8 \%)$ & .0004 \\
\hline Lowest hemoglobin & $88(75.5-100)$ & $83.5(71.5-91)$ & $<.0001$ \\
\hline \multicolumn{4}{|l|}{ Postoperative characteristics } \\
\hline Total ventilation time & $3.3(0-8.0)$ & $10.4(2.3-41)$ & $<.0001$ \\
\hline Mechanical ventilation $>24 \mathrm{~h}$ & $42(4.9 \%)$ & $43(29.1 \%)$ & $<.0001$ \\
\hline Return to operating room & $72(8.2 \%)$ & $35(23.0 \%)$ & $<.0001$ \\
\hline Inotrope/vasopressor/IABP $>24 \mathrm{~h}$ & $131(15.1 \%)$ & $53(35.1 \%)$ & $<.0001$ \\
\hline Atrial fibrillation & $310(35.4 \%)$ & $77(50.7 \%)$ & .0003 \\
\hline Ventricular arrhythmia & $230(26.3 \%)$ & $65(43.1 \%)$ & $<.0001$ \\
\hline Dialysis (renal failure) & $4(0.5 \%)$ & $7(4.6 \%)$ & .0003 \\
\hline Renal insufficiency ( $>50 \%$ increase from baseline creatinine level) & $56(6.4 \%)$ & $34(22.4 \%)$ & $<.0001$ \\
\hline Stroke/TIA & $4(0.5 \%)$ & $10(6.8 \%)$ & $<.0001$ \\
\hline Infection & $117(13.4 \%)$ & $46(30.3 \%)$ & $<.0001$ \\
\hline Sepsis & $6(0.7 \%)$ & $5(3.3 \%)$ & .0146 \\
\hline Lowest sodium & $134(131-135)$ & $133(130-135)$ & .0127 \\
\hline Highest creatinine & $88(74-110)$ & $108(87-150)$ & $<.0001$ \\
\hline $\mathrm{RBC}$ transfusion & $370(42.3 \%)$ & $104(68.4 \%)$ & $<.0001$ \\
\hline FFP transfusion & $195(22.3 \%)$ & $70(46.1 \%)$ & $<.0001$ \\
\hline Platelet transfusion & $197(22.5 \%)$ & $66(43.4 \%)$ & $<.0001$ \\
\hline Any blood transfusion $\dagger$ & $446(50.9 \%)$ & $119(78.3 \%)$ & $<.0001$ \\
\hline Cryoprecipitate & $5(0.6 \%)$ & $4(2.6 \%)$ & .0318 \\
\hline
\end{tabular}

Data presented as $\mathrm{n}(\%)$ for categorical variables and mean \pm standard deviation or median (25th percentile-75th percentile) for continuous variables. $C A B G$, Coronary artery bypass grafting; $I A B P$, intra-aortic balloon pump; $T I A$, transient ischemic attack; $R B C$, red blood cell; $F F P$, fresh frozen plasma. *Not a statistically significant univariate risk factor but below threshold of $P<.20$ and therefore included in multivariate analysis. †Included at least 1 of RBC, FFP, or platelet transfusions.

indicate that the mechanism of delirium differs across age groups and reveals, more broadly, that strategies for delirium prevention might require group-specific interventions.

Concomitant CABG and valve surgery was associated with the occurrence of in-hospital delirium, a finding previously reported. ${ }^{16,17}$ This might be attributed to iatrogenic factors related to a longer and more complex surgery. However, in our population, the cardiopulmonary bypass time, surgery duration, and intraoperative blood transfusion were not identified as independent risk factors 
TABLE 3. Multivariate analyses of risk factors associated with in-hospital delirium after cardiac surgery

\begin{tabular}{|c|c|c|c|}
\hline Risk factor & OR & $95 \%$ CI & $\begin{array}{c}P \\
\text { value }\end{array}$ \\
\hline \multicolumn{4}{|l|}{ Preoperative characteristics } \\
\hline Age $\geq 65$ y & 3.21 & $2.02-5.10$ & $<.001$ \\
\hline Prehospital admission benzodiazepine use & 2.11 & $1.09-4.07$ & .03 \\
\hline \multicolumn{4}{|l|}{ Intraoperative characteristics } \\
\hline CABG and valve procedure & 2.30 & $1.36-3.88$ & .002 \\
\hline \multicolumn{4}{|l|}{ Postoperative characteristics } \\
\hline Stroke/TIA & 6.03 & $1.69-21.53$ & .006 \\
\hline Mechanical ventilation $>24 \mathrm{~h}$ & 4.34 & $2.52-7.45$ & $<.001$ \\
\hline Any blood transfusion & 2.02 & $1.30-3.15$ & .002 \\
\hline $\begin{array}{l}\text { Renal insufficiency ( }>50 \% \text { increase from } \\
\text { baseline creatinine level) }\end{array}$ & 1.91 & $1.08-3.37$ & .03 \\
\hline
\end{tabular}

$\overline{O R}$, Odds ratio; $C I$, confidence interval; $C A B G$, coronary artery bypass grafting; $T I A$, transient ischemic attack.

associated with the development of delirium. An alternate explanation could be that patients undergoing this type of surgery are older and generally have a greater comorbidity burden than those undergoing isolated CABG or valve surgery. Given that age was an independent risk factor for delirium, a comparison of age was done within the delirium subset to determine whether this factor was more pronounced in patients undergoing this type of surgery. No significant difference was found between the mean age of the patients undergoing concomitant CABG and valve surgery who developed delirium and other patients with delirium (data not shown).

Prehospital admission benzodiazepine use was relatively low in our population $(7.4 \%)$; however, approximately $20 \%$ of patients taking benzodiazepines subsequently developed delirium after their cardiac surgery. An abrupt reduction or discontinuation of these drugs could have led to withdrawal symptoms, which can include confusion and delirium. ${ }^{18}$ Pisani and colleagues ${ }^{19}$ reported that benzodiazepine use was associated with the duration of ICU delirium. In our study population, if we attributed every case of delirium involving benzodiazepine use to withdrawal, this would still only represent a small percentage of the total number of cases $(10.6 \%)$. This provides further support to the idea that this syndrome is multifactorial in origin. Data on the preoperative use of other psychiatric medications such as antidepressants and antipsychotics were collected and were not found to be associated with the development of delirium. Furthermore, data on documented or self-reported preoperative dementia were captured and not found to be associated with delirium in the present study, although the patient numbers were low.

The receipt of any blood product transfusion postoperatively was associated with in-hospital delirium, consistent with previous findings. ${ }^{15,16}$ Furthermore, it has been previously reported that blood transfusion was associated with increased mortality after cardiac surgery. ${ }^{20}$ However, the exact mechanism of how blood transfusion contributes to delirium and other adverse outcomes has not been well characterized. It is possible that medications administered concurrently with postoperative transfusions could have confounded the association between blood products and delirium; therefore, the latter relationship might an example of confounding by indication.

In our study population, new renal insufficiency in the postoperative period, which was defined as a greater than $50 \%$ increase from the baseline creatinine level, was a risk factor associated with in-hospital delirium. This has been corroborated by previous studies, ${ }^{19,21}$ which found elevated creatinine levels to be predictive of delirium. Acute kidney injury associated with cardiac surgery has been reported to range from $5 \%$ to $10 \%$ and has been associated with adverse outcomes, ${ }^{22}$ including 30-day mortality. ${ }^{23}$ In our study population, the overall rate of new renal insufficiency was $9.9 \%(\mathrm{n}=100)$. In contrast, the rate was more than double in patients with delirium $(22.4 \%$; odds ratio, $1.91 ; P=.03$ ). This suggests that the inflammation and hemodynamic perturbation of cardiac surgery that often leads to acute kidney injury might share a mechanism of pathogenesis with delirium. ${ }^{24}$

\section{Study Limitations}

The present study had several limitations worth noting. First, this was a single-center study, which might limit its generalizability. However, the large sample size and patient characteristics of our study cohort were similar to those of previously reported contemporary surgical cohorts. Second, the lack of temporal information for the postoperative variables other than the prevalence of delirium constrained our ability to determine associations between many of the postoperative risk factors and the onset of delirium. Third, the sedation and agitation level data, assessed through use of the RASS, was collected multiple times each day in the ICU for a given patient but not consistently synchronous with the CAM assessments. Therefore, associations between the risk factors identified and delirium motor subtypes (ie, hypoactive, hyperactive, mixed subtype) could not be made. Finally, ours was a retrospective analysis; thus, preoperative cognitive testing data were not available because it was not formally performed during the study period. Recently, Saczynski and colleagues ${ }^{25}$ demonstrated that delirium was more common in patients with lower preoperative minimental status examination scores. Future studies are planned for a more detailed examination of the effect of pre-existing cognitive and functional impairment on postoperative delirium.

\section{CONCLUSIONS}

Delirium is a common complication in patients undergoing cardiac surgery. The prevalence of delirium was studied 
in a cardiac surgery patient population in which the ICU environment was the primary factor changed. Although the ICU environment did not have a significant effect on the overall prevalence of delirium, that does not preclude the possibility that the ICU environment might interact with other factors, such as age, in a complex manner. We have identified other risk factors associated with in-hospital delirium after cardiac surgery. Validation of these risk factors and additional investigation of the preoperative risk factors are needed. In developing preventative strategies and interventional protocols for addressing delirium, adjusting the ICU environment in isolation will likely not be sufficient and instead will require a more comprehensive multimodal approach.

\section{References}

1. Ely E, Gautam S, Margolin R, Francis J, May L, Speroff T, et al. The impact of delirium in the intensive care unit on hospital length of stay. Intens Care Med. 2001;27:1892-900.

2. Milbrandt EB, Deppen S, Harrison PL, Shintani AK, Speroff T, Stiles RA, et al. Costs associated with delirium in mechanically ventilated patients. Crit Care Med. 2004;32:955-62.

3. Ely EW, Shintani A, Truman B, Speroff T, Gordon SM, Harrell FE Jr, et al. Delirium as a predictor of mortality in mechanically ventilated patients in the intensive care unit. JAMA. 2004;291:1753-62.

4. Baillot RG, Joanisse DR, Stevens LM, Doyle DP, Dionne B, Lellouche F. Recent evolution in demographic and clinical characteristics and in-hospital morbidity in patients undergoing coronary surgery. Can J Surg. 2009;52:394-400.

5. Martin B-J, Buth KJ, Arora RC, Baskett RJF. Delirium: a cause for concern beyond the immediate postoperative period. Ann Thorac Surg. 2012;93:1114-20.

6. Inouye SK, van Dyck CH, Alessi CA, Balkin S, Siegal AP, Horwitz RI. Clarifying confusion: the confusion assessment method. A new method for detection of delirium. Ann Intern Med. 1990;113:941-8.

7. Ely EW, Margolin R, Francis J, May L, Truman B, Dittus R, et al. Evaluation of delirium in critically ill patients: validation of the Confusion Assessment Method for the intensive care unit (CAM-ICU). Crit Care Med. 2001;29:1370-9.

8. Sessler CN, Gosnell MS, Grap MJ, Brophy GM, O’Neal PV, Keane KA, et al. The Richmond Agitation-Sedation Scale. Am J Respir Crit Care Med. 2002; 166:1338-44.

9. Wenham T, Pittard A. Intensive care unit environment. Contin Educ Anaesth Crit Care Pain. 2009;9:178-83.
10. Flaherty JH, Little MO. Matching the environment to patients with delirium: lessons learned from the delirium room, a restraint-free environment for older hospitalized adults with delirium. J Am Geriatr Soc. 2011;59:S295-300.

11. Kumar K, Zarychanski R, Bell DD, Manji R, Zivot J, Menkis AH, et al. Impact of 24-hour in-house intensivists on a dedicated cardiac surgery intensive care unit. Ann Thorac Surg. 2009;88:1153-61.

12. Koster S, Hensens AG, Schuurmans MJ, van der Palen J. Risk factors of delirium after cardiac surgery: a systematic review. Eur J Cardiovasc Nurs. 2011;10:197-204.

13. Gottesman RF, Grega MA, Bailey MM, Pham LD, Zeger SL, Baumgartner WA, et al. Delirium after coronary artery bypass graft surgery and late mortality. Ann Neurol. 2010;67:338-44.

14. Kazmierski J, Kowman M, Banach M, Fendler W, Okonski P, Banys A, et al. Incidence and predictors of delirium after cardiac surgery: results from the IPDACS Study. J Psychosom Res. 2010;69:179-85.

15. Stransky M, Schmidt C, Ganslmeier P, Grossmann E, Haneya A, Moritz S, et al. Hypoactive delirium after cardiac surgery as an independent risk factor for prolonged mechanical ventilation. J Cardiothor Vasc Anesth. 2011;25:968-74.

16. Bucerius J, Gummert JF, Borger MA, Walther T, Doll N, Falk V, et al. Predictors of delirium after cardiac surgery delirium: effect of beating-heart (off-pump) surgery. J Thorac Cardiovasc Surg. 2004;127:57-64.

17. Katznelson R, Djaiani GN, Borger MA, Friedman Z, Abbey SE, Fedorko L, et al. Preoperative use of statins is associated with reduced early delirium rates after cardiac surgery. Anesthesiology. 2009;110:67-73.

18. Ashton $\mathrm{H}$. The diagnosis and management of benzodiazepine dependence. Curr Opin Psychiatry. 2005;18:249-55.

19. Pisani MA, Murphy TE, Araujo KLB, Slattum P, Van Ness PH, Inouye SK. Benzodiazepine and opioid use and the duration of intensive care unit delirium in an older population. Crit Care Med. 2009;37:177-83.

20. Paone G, Brewer R, Theurer PF, Bell GF, Cogan CM, Prager RL. Preoperative predicted risk does not fully explain the association between red blood cell transfusion and mortality in coronary artery bypass grafting. $J$ Thorac Cardiovasc Surg. 2012;143:178-85.

21. Bakker RC, Osse RJ, Tulen JHM, Kappetein AP, Bogers AJJC. Preoperative and operative predictors of delirium after cardiac surgery in elderly patients. Eur J Cardiothorac Surg. 2012;41:544-9.

22. Shaw A, Swaminathan M, Stafford-Smith M. Cardiac surgery-associated acute kidney injury: putting together the pieces of the puzzle. Nephron Physiol. 2008;109:55-60.

23. Mitter N, Shah A, Yuh D, Dodd O-J, Thompson RE, Cameron D, et al. Renal injury is associated with operative mortality after cardiac surgery for women and men. J Thorac Cardiovasc Surg. 2010;140:1367-73.

24. Rudolph JL, Ramlawi B, Kuchel GA, McElhaney JE, Xie D, Sellke FW, et al. Chemokines are associated with delirium after cardiac surgery. J Gerontol Ser A: Biol Sci Med Sci. 2008;63:184-9.

25. Saczynski JS, Marcantonio ER, Quach L, Fong TG, Gross A, Inouye SK, et al. Cognitive trajectories after postoperative delirium. N Engl J Med. 2012;367: $30-9$. 